\title{
Entrepreneurship
}

Jan a Jun $2021-$ v.5 - n.1

ISSN: 2595-4318

This article is also available online at: www.sustenere.co

\section{Cenário e perspectiva da criptomoeda bitcoin no Brasil}

Este artigo tem o propósito de compreender questões pertinentes a respeito do contexto econômico da Criptomoeda Bitcoin, tanto no cenário mundial quanto no cenário nacional. Levando em consideração dados históricos e projeções futuras, fazendo também, uma análise fundamentalista dos princípios de tal Criptomoeda. O estudo em questão busca mostrar uma nova forma de investimento para pessoas que buscam potencializar seus rendimentos financeiros através de renda variável, ou seja, de um investimento que aproveita a volatilidade do mercado para adquirir rentabilização. Além do exposto, este estudo também aborda os conceitos voltados a compreensão do Bitcoin e das Criptomoedas, trazendo uma relação com a economia tradicional e desvendando qual é o público-alvo desse novo mercado, além de assimilar os principais motivos pelos quais esse público faz essas aplicações. A metodologia utilizada no desenvolvimento deste trabalho foi a partir da pesquisa bibliográfica e web bibliográfica.

Palavras-chave: Bitcoin; Criptomoeda; Economia; Investimento; Renda variável.

\section{Scenario and perspective of bitcoin cryptocurrency in Brazil}

This article aims to understand pertinent issues regarding the economic context of Bitcoin Cryptocurrency, both on the world stage and on the national stage. Taking into account historical data and future projections, also making a fundamental analysis of the principles of such Cryptocurrency. The study in question seeks to show a new form of investment for people who seek to enhance their financial income through variable income, that is, an investment that takes advantage of market volatility to gain profitability. In addition to the above, this study also addresses concepts aimed at understanding Bitcoin and Cryptocurrencies, bringing a relationship with the traditional economy and unraveling who is the target audience of this new market, in addition to assimilating the main reasons why this audience makes these applications. The methodology used in the development of this work was based on bibliographic research and bibliographic web.

Keywords: Bitcoin; Cryptocurrency; Economy; Investment; Variable income.

Topic: Finanças Empresariais

Reviewed anonymously in the process of blind peer.
Received: $20 / 02 / 2021$

Approved: 22/05/2021
Genisson dos Santos Pinheiro (iD)

Faculdade do Nordeste da Bahia, Brasil http://lattes.cnpq.br/1028165517527785 http://orcid.org/0000-0002-9674-4113

genissonpinheiro@meumoney.info

Alvani Bomfim de Sousa Júnior

Faculdade do Nordeste da Bahia, Brasil

http://lattes.cnpq.br/6358502728889050

http://orcid.org/0000-0002-8714-4175

alvanijunior@yahoo.com.br

Josivan dos Santos Moura

Faculdade do Nordeste da Bahia, Brasil

http://lattes.cnpq.br/6358502728889050

http://orcid.org/0000-0002-1693-5164

josivan@gmail.com 


\section{INTRODUÇÃO}

Este trabalho tem como objetivo compreender o cenário e perspectiva da Criptomoeda Bitcoin no Brasil. Por se tratar de uma moeda digital e estarmos situados em um modelo de sociedade tecnológica ancorada na influência das Tecnologias da Informação e Comunicação (TIC) na vida econômica das pessoas, cada vez mais, encontramos investidores que buscam potencializar e diversificar suas aplicações através desse tipo de investimento, com isso surge a necessidade de debater acerca de tal assunto.

Nesse contexto, é evidente o crescimento mundial dos investimentos da Criptomoeda Bitcoin, segundo informações levantadas pelo provedor de dados de Criptomoedas, Coinmetrics, mais de 2,7 milhões de endereços possuem mais de 0,1 Bitcoin em 2019 (o que equivale a $\mathrm{R} \$ 3.355,00$ na cotação atual), o que representa um aumento de 58\% em relação a 2017 (PORTAL DO BITCOIN, 2019). A expansão desse mercado e as possibilidades de ganhos mais elevados (por se tratar de um investimento de renda variável, ou seja, não há nenhum valor de rendimento predeterminado), faz com que esse tipo de investimento seja uma alternativa bastante procurada pelos investidores mais agressivos.

Tais fatores justificam ter a Criptomoeda Bitcoin como sendo um dos assuntos importantes para serem debatidos, investigados, estudados por pesquisadores, gestores, professores e alunos na contemporaneidade. Assim, como aluno do último período do curso de Administração de Empresas tive o interesse de realizar uma pesquisa bibliográfica e web bibliográfica tendo como objeto de investigação a Criptomoeda Bitcoin. A intenção é fazermos uma investigação que além de usar livros, artigos etc. a internet também seja um recurso de obtenção de dados. A intenção é que ambas possam revelar o cenário e perspectiva da Criptomoeda Bitcoin no Brasil e no mundo, levando em consideração a forma de operacionalização e conhecimento que esse tipo de investimento requer das pessoas sob o ponto de vista administrativo.

\section{DISCUSSÃO TEÓRICA}

\section{O que dizer sobre criptomoedas e bitcoin?}

Nos últimos 10 anos ouvimos falar de questões relacionadas à Criptomoedas e ao seu mercado econômico, seja em jornais televisivos ou em matérias de sites e blogs. Conforme a ideia da Comissão dos Valores Mobiliários (CVM), Criptomoedas são códigos virtuais que funcionam como forma de dinheiro na internet, porém é possível comprar produtos físicos com esse sistema de troca (CVM, 2018). No dia 31 de outubro de 2008 o projeto do Bitcoin foi publicado pelo anônimo Satoshi Nakamoto.

Nesse período vivenciava-se a crise Subprime ${ }^{1}$, e como o dólar estava desvalorizado, essa moeda digital pôde se enquadrar no contexto de uma possível alternativa de reserva de valor em situações como aquela, de crises financeiras. No atual sistema monetário mundial existe uma moeda estatal denominada de moeda fiduciária, como existe uma regulamentação e distribuição por parte do Governo é uma moeda

${ }^{1}$ A crise do subprime, chamada por muitos de "bolha imobiliária americana", teve seu início a partir da forte queda do índice Dow Jones em julho de 2007, motivada pela hipótese do colapso hipotecário, que arrastou várias instituições financeiras americanas para a situação de insolvência. Disponívelem:www.sunoresearch.com.br/artigos/crise-do-subprime/>. Acesso em:15 set. 2019. 
obrigatoriamente centralizada. Ao contrário do Bitcoin que é descentralizado, isso acontece porque ele não é dependente de um órgão específico para funcionar, como explica Bitcoin (2015):

Novos bitcoins são gerados através de um processo competitivo e descentralizado chamado "mineração". Esse processo consiste na recompensa dada aos usuários pelos seus serviços. Os "mineiros" de Bitcoin estão processando transações e fazendo a rede segura usando hardware especializado e coletando novos bitcoins em troca.

Ou seja, o funcionamento da moeda em si não necessita de centralização, porém, existem empresas que negociam Bitcoins, essas sim são empresas centralizadas e funcionam como qualquer outra empresa do ponto de vista organizacional, com níveis: estratégico, tático e operacional.

O Bitcoin não surgiu como uma forma de investimento, o seu objetivo era ser uma moeda mundial, que realizasse transações entre países de forma rápida e sem a existência de taxas altas de câmbio ou de negociação, além disso, o mesmo também prezava pela lei da oferta e demanda, onde o próprio mercado impulsiona ou regride o preço sem a influência governamental, como salienta Ulrich (2014) "o Bitcoin é uma forma de dinheiro, assim como o real, o dólar ou o euro, com a diferença de ser puramente digital e não ser emitido por nenhum Governo". O fato dessa Criptomoeda em específico não ter um órgão regulador atrelado, não significa que as transações sejam fraudulentas ou proporcionem prejuízos aos investidores. Deve-se isso à rede Blockchain ${ }^{2}$, que registra todas as transações e as validam.

Essa moeda digital obteve uma aceitação elevada. Atrelado ao nível de aceitação e negociação, o preço dessa Criptomoeda saiu de menos de U\$100 em janeiro de 2012 para mais de U\$19.000 em dezembro de 2017, como mostra o gráfico da Bitstamp, empresa que negocia moedas digitais.

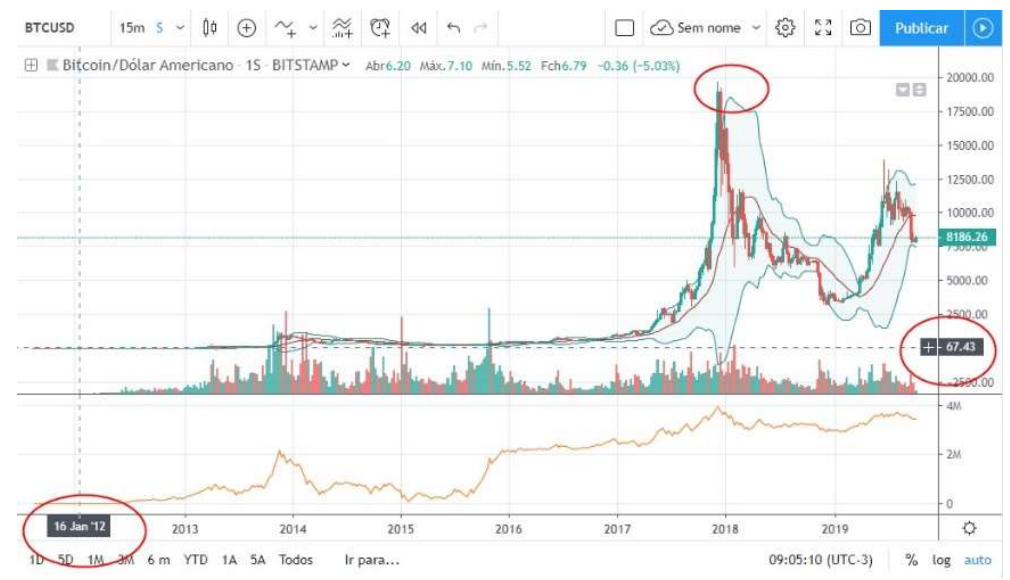

Gráfico 1: Preço do Bitcoin ao longo dos anos ${ }^{3}$.

No Gráfico 1 acima é perceptível ver o aumento no preço do Bitcoin ao longo dos anos. Ainda relacionado com o conceito introdutório às Criptomoedas, temos o entendimento de Gustavo Rocha: Para ele as Criptomoedas são da espécie das moedas digitais, são ferramentas monetárias, mas com um diferencial, são criptografadas (ROCHA, 2019).

O autor traz informações simplificadas e de fácil entendimento sobre a conceituação de

${ }^{2} \mathrm{O}$ blockchain é um banco de dados. O que o faz tão importante é o fato de esse não ser um banco de dados convencional: o sistema funciona como um livro de registros, mas inviolável, "inderrubável" e extremamente eficiente. Disponível em: <https://www.infowester.com/blockchain.php> Acesso em:15 set. 2019.

${ }^{3}$ https://br.tradingview.com/chart/LyDV8Ar3/ 
Criptomoeda, e ainda aborda uma questão muito relevante, ao dizer que "as Criptomoedas são da espécie das moedas digitais", isso porque existem outros tipos de moedas digitais, como por exemplo o dinheiro que é pago quando se usa o cartão de crédito, é algo intangível, você não tem necessariamente acesso às cédulas.

\section{Cenário econômico do bitcoin no mundo}

O Bitcoin se insere cada vez mais no contexto de investimento. As grandes variações de preços que este mercado apresenta acaba atraindo novos investidores e adeptos. O aumento no interesse pelas Criptomoedas é evidenciado pelo Google, o qual traz dados muito relevantes, que colocam à procura pelo termo "Bitcoin" maior que pelo termo "Stocks" (ações) (FERREIRA, 2019). Além da grande volatilidade que este mercado possui, outro fator que o coloca à frente do mercado de ações no interesse de investidores, é a não necessidade de investimentos muito altos, desta forma, pessoas com menor poder aquisitivo podem fazer aportes. É possível comprar $\mathrm{R} \$ 1$ de Bitcoin, por exemplo. Neste aspecto socioeconômico, Fernando Ulrich afirma que o Bitcoin tem o potencial de melhorar a qualidade de vida das classes mais pobres do mundo, o mesmo cita uma estatística de que $64 \%$ da população que vive em países em desenvolvimento não têm acesso a serviços financeiros básicos, isso porque, para instituições financeiras serem implantadas em lugares de baixa renda, é inviável. Entretanto, o Bitcoin possibilita o acesso financeiro em qualquer parte do mundo, desde que o indivíduo esteja conectado à internet (ULRICH, 2014).

Para complementar a ideia de Ulrich, temos uma relação de aceitação do Bitcoin ao longo dos últimos 6 anos. É notório que, até mesmo em algumas ilhas como o Hawaii, dentre outras, às Criptomoedas são aceitas, conforme as imagens abaixo nos revelam. Nesse contexto, a moeda digital Bitcoin pode ser um bom meio de troca para turistas, já que os mesmos poderão viajar por diferentes partes do mundo sem a necessidade de conversão de valor para a moeda local.

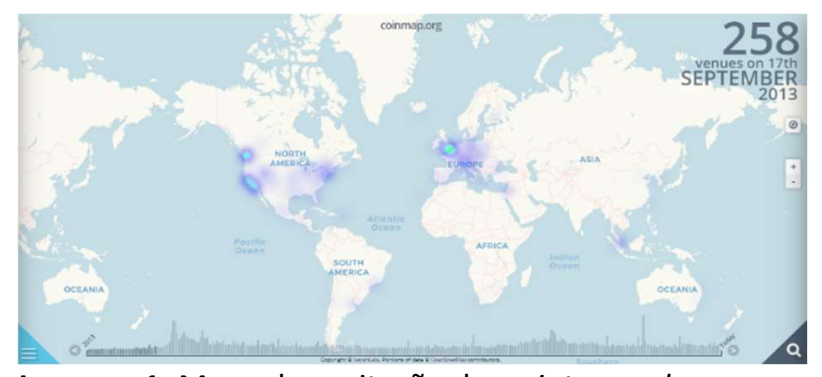

Imagem 1: Mapa da aceitação das criptomoedas no ano de $2013^{4}$.

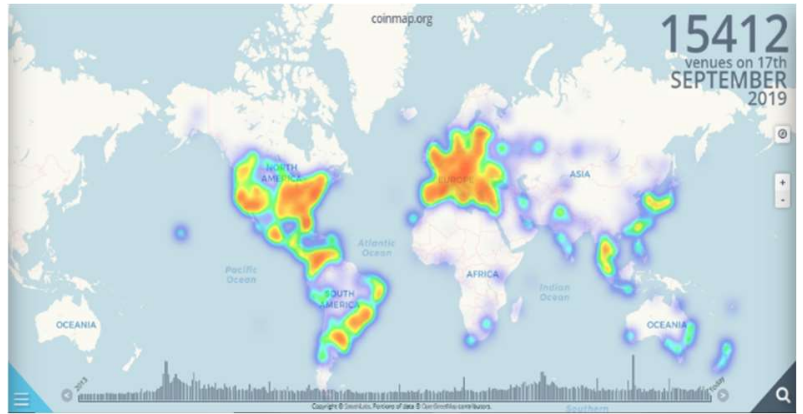

Imagem 2: Mapa da aceitação das criptomoedas no ano de $2019^{5}$.

Ao longo desses últimos dez anos, o mercado de Criptomoedas vem ganhando reconhecimento e aceitação. De acordo com o CoinMap, ferramenta que mensura a aceitação das criptomoedas de forma global, em setembro de 2013 apenas 258 estabelecimentos aceitavam pagamentos por esse meio, fazendo uma relação de 2013 com os números atuais que são de 15.412, totaliza-se um aumento de cerca de 5.873\%, como mostra a imagem acima: é perceptível também, que a aceitação com maior volume se iniciou na área

\footnotetext{
${ }^{4}$ https://coinmap.org/\#/world/48.10743119/-203.55468750/2/

${ }^{5}$ https://coinmap.org/\#/world/48.10743119/-203.55468750/2/ 
que é localizada a Europa, isto foi justamente porque neste local surgiu a primeira Exchange, que é o nome dado às casas de câmbio de Criptomoedas. Em primeiro momento, a empresa denominada de Bitcoin Market, e criada por Dwdollar, tinha a ideia de unir pessoas que queriam comprar Bitcoin, com pessoas que desejavam vender, esta empresa em específico não funciona atualmente, mas diversas outras surgiram (HENRIQUE, 2019).

Da perspectiva de aceitação, o nosso objeto de pesquisa vem se mostrando como sendo um dos interesses de grandes investidores e visionários, como é o exemplo de Elon Musk CEO da Tesla Motors, empresa que produz carros elétricos, a organização já declarou abertamente que vendeu um Tesla Model S com pagamento em Bitcoin, a transação foi de mais de R\$700.000,00 (BASILIO, 2017). Com tal informação fica mais claro perceber que grandes empreendimentos já buscam a atualização e adequação às novas tecnologias.

O Bitcoin no contexto de investimento funciona com um princípio que acaba agregando valor, o princípio de escassez. O seu projeto visa que serão postos no mercado 21 milhões de moedas digitais, o que vai o tornando raro com o passar dos anos, já que mais pessoas vão aderindo moedas e dessa forma, menos moedas ficam em circulação. Atualmente, o valor de mercado ${ }^{6}$ dessa Criptomoeda é de mais de 600 bilhões de reais, como mostra a ferramenta de volume de negociações Coin Market Cap.

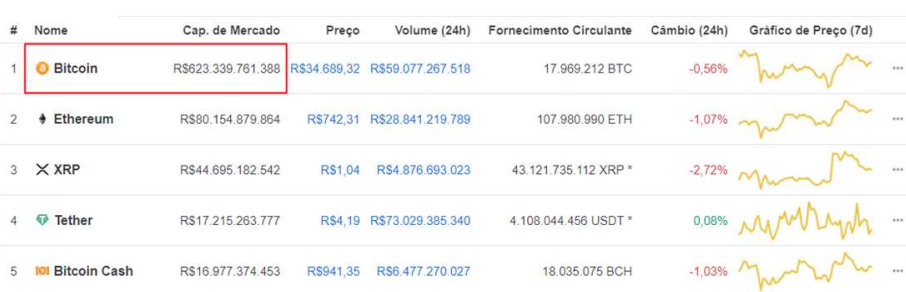

Imagem 3: Valor de mercado das 5 maiores Criptomoedas ${ }^{7}$.

No cenário atual o Bitcoin assume a colocação de maior Criptomoeda com base em seu valor de mercado, o que a coloca a mais de 540 bilhões de reais à frente do segundo colocado, Ethereum, com tal informação também fica entendido que cada projeto de Criptomoeda têm suas peculiaridades, desta forma, o mercado aceita às mesmas de maneiras diferentes e isso causa grandes diferenças de preços.

Ainda a respeito da imagem 3, é importante pautar que o "Fornecimento Circulante" no momento é de pouco menos de 18 milhões de Bitcoins, isso significa que pouco mais de 3 milhões ainda serão minerados pelos usuários. $O$ fato de existir um número limitado de moedas que serão fornecidas agrega bastante na formação do seu preço. Segundo dados do World Wealth Report 2019, que foi publicado pelo site Folha de São Paulo, em 2018 existiam 18 milhões de milionários no mundo (CAGLIARI, 2019), ou seja, em uma suposta situação se cada um deles comprassem 1 Bitcoin não existiriam mais Criptomoedas deste tipo sendo ofertadas, e isso possivelmente resultaria em um aumento do preço, desta forma fica mais visível o impacto econômico de um ativo que possui limite de emissão.

\footnotetext{
${ }^{6}$ O valor de mercado é o resultado da multiplicação do valor atual da ação de uma companhia pelo número de ações existentes. Disponível em: https://maisretorno.com/blog/termos/v/valor-de-mercado Acesso em: 01 out. 2019.

${ }^{7}$ https://coinmarketcap.com/pt-br/
} 


\section{O bitcoin no contexto nacional}

É visível que a imagem 2 mostra um aumento no número de aceitação de Criptomoedas no território nacional. Isso demonstra que o brasileiro está buscando novas formas de investimentos e as empresas novas formas de receber seus pagamentos, para complementar este raciocínio temos dados relevantes da Global Digital Report que foram divulgados pelo site brasileiro CriptoFacil, a pesquisa aponta o Brasil como sendo o $5^{\circ}$ país do mundo com mais usuários de Criptomoedas, a mesma diz que: "cerca de 8,1\% dos brasileiros entre 16 e 64 anos possui alguma criptomoeda" (GUSSON, 2019).

No Brasil, tanto algumas empresas virtuais como também físicas, já aceitam este meio de pagamento. No segmento online temos o exemplo da Hostinger, que é uma organização que negocia hospedagens e domínios para sites (NUNES, 2019). Na categoria de empresas físicas exemplifica-se com o Shopping Paço Alfândega, localizado na cidade de Recife, um dos representantes do mesmo afirma que:

Essa é a moeda do futuro e nosso conceito é ser o shopping do futuro. Não tem uma região melhor em Pernambuco para iniciar essa experiência do que no Bairro do Recife, onde existe o maior polo tecnológico da América Latina, que é o Porto Digital. (AZEVEDO, 2017)

Isso mostra que as empresas vêm se moldando para buscar diferenciais e oportunidades, e o Bitcoin acaba sendo um atrativo tanto do ponto de vista de economia, (já que as taxas são inferiores às de cartões de crédito por exemplo) quanto da óptica de adaptações às novas tecnologias, sem contar na segurança proporcionada, já que desta forma o indivíduo acaba deixando de andar com dinheiro em espécie.

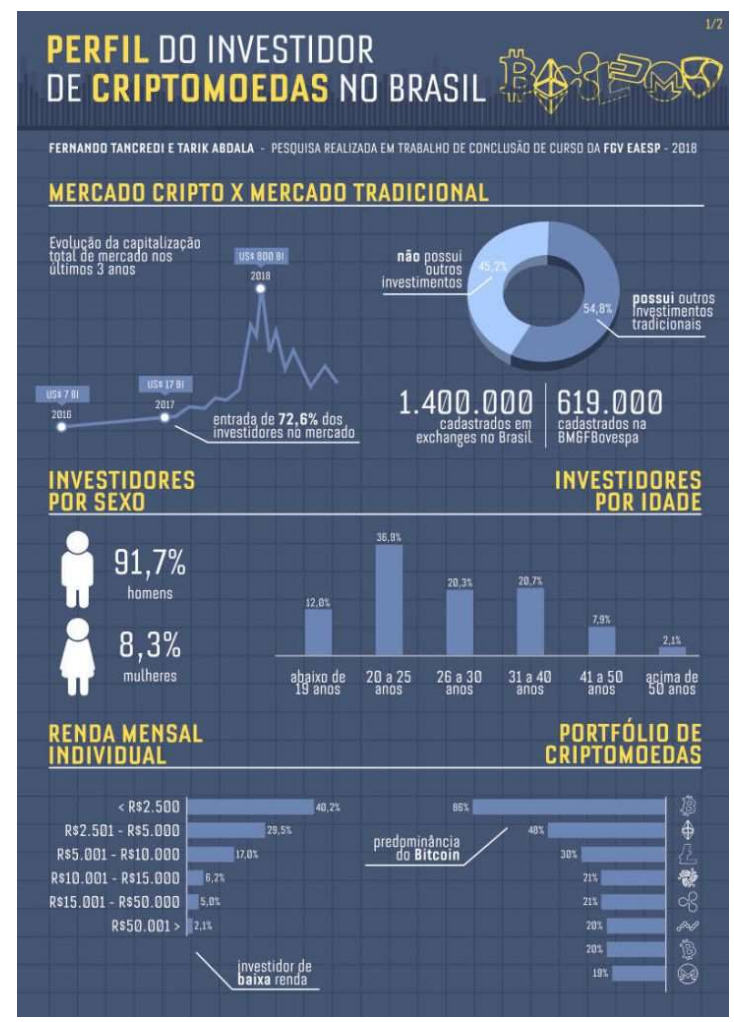

Imagem 4: Perfil do investidor brasileiro de Criptomoedas ${ }^{8}$.

Visto o mercado em ascensão e às possíveis oportunidades que o mesmo pode oferecer, cada vez mais empresários resolvem criar empresas destinadas para compra e venda de Criptomoedas, "no Brasil,

${ }^{8}$ https://cointimes.com.br/quem-e-o-investidor-brasileiro-de-criptomoedas/ 
existem mais de 30 Exchanges de Criptomoedas para investidores negociarem bitcoins e outras moedas" (INFOCHAIN, 2019). Com o aumento de novas empresas do ramo, algo positivo se reflete no mercado do Bitcoin, que é a liquidez, ou seja, a facilidade em transformar o ativo digital em moeda fiduciária, no nosso caso, em real. Para nos situarmos melhor sobre o atual cenário do Bitcoin no Brasil é essencial fazermos abordagens à dados significativos, informações essas que demonstrem o interesse desse mercado pela população brasileira, da mesma forma é muito relevante apontarmos quem são os brasileiros que optam por esse segmento de mercado e o porquê. O relatório foi divulgado e comentado pelo site CoinTimes, o qual também desenvolveu a imagem 4 explica.

Pela representação gráfica acima as pessoas que mais investem em Criptomoedas no Brasil são homens, com uma renda mensal considerada como baixa e além de tudo isso, a maioria são jovens de até 30 anos. Para John Coates, um especialista em neurologia da Universidade de Cambridge, no Reino Unido, em geral os homens costumam fazer investimentos de maior risco, almejando transações bem-sucedidas e mais lucrativas (BBC, 2018). Ainda sobre as estatísticas da imagem 5, o próprio site faz colocações muito pertinentes:

Mais de $90 \%$ dos investidores são homens, e sua média de idade é de 28 anos. Cerca de $57,2 \%$ dos investidores têm entre 20 e 30 anos, e apenas $10 \%$ deles possuem mais de 41 anos. $\mathrm{Na} \mathrm{B} 3$, a bolsa de valores paulista, a média de idade é bem maior: $80,7 \%$ dos investidores cadastrados têm acima de 36 anos. (TANCREDI, 2018)

Com esses dados é possível perceber que as pessoas de idade mais elevada não são muito adeptas do Bitcoin. Possivelmente isso está atrelado ao fato de ser um mercado consideravelmente recente, e que acaba trazendo conceitos bastante diferentes, como por exemplo ter o seu dinheiro convertido em uma moeda digital. Com isso, pode haver uma certa resistência na adaptação. Outro ponto que precisa ser relacionado é o de investidores de Criptomoedas com os investidores da Bolsa de Valores, os dados apontam que em janeiro de 2018 os investidores de Bitcoin e outras moedas digitais já representavam mais que o dobro da quantidade de pessoas que fazem aplicações na $\mathrm{B}^{9}{ }^{9}$, eram 1,4 milhão de usuários cadastrados nas 3 maiores casas de câmbio de Criptomoedas do Brasil, a Foxbit, BitcoinToYou e Mercado Bitcoin, enquanto a Bolsa de Valores brasileira contava com 619 mil pessoas cadastradas (SIMÕES et al., 2018).

Isso demonstra que mesmo o investimento em ações sendo mais antigo, o mercado de Criptomoedas vem ganhando cada vez mais usuários e superando mercados que existem há décadas.

\section{CONCLUSÕES}

Com base nos dados e análises aqui mencionados podemos tirar algumas conclusões a respeito da Criptomoeda Bitcoin, respostas essas que serão resumidas e expostas. Mesmo se tratando de uma tecnologia econômica e consideravelmente recente, a mesma já tem um vasto mercado, cada vez mais empresas e usuários se adéquam ao uso das moedas Criptografadas e, principalmente, ao Bitcoin. Com base no princípio da escassez mencionado anteriormente a perspectiva para o preço do Bitcoin nos próximos anos é de alta, 
claro que se tratando de um ativo muito volátil, o cenário pode se reverter, porém com a grande quantidade de aceitação que essa moeda digital vem adquirindo a probabilidade de que o preço se sustente é maior.

A faixa etária que mais se adequa às Criptomoedas, são os jovens de até 30 anos de idade, os dados mostram que quanto mais idade menos adeptos ao Bitcoin e afins eles são. Isso ocorre devido ao fato de ser um conceito recente e é normal que haja uma certa resistência do público mais idoso. Ainda sobre a população que mais investe nesse segmento, vale ressaltar que com base nos dados deste estudo, os homens representam a maior parte do mercado, isso porque o extinto mais agressivo nas aplicações é bastante presente nos mesmos, em geral as mulheres são mais conservadoras. Tendo em mente toda a volatilidade desse mercado, o Bitcoin não é um bom ativo para uma reserva de emergência, revelando-se, dessa forma, um investimento de risco, como por exemplo à estabilidade financeira.

Porém, o mesmo pode ser uma boa opção para criação de reserva de valor, ou seja, visando o longo prazo e evitando a inflação que dilui o preço do real. Do ponto de vista dos investimentos o Bitcoin pode ser uma boa alternativa, principalmente para os investidores mais engajados, os que optam por um pouco mais de risco e a possibilidade de obter mais retorno em curto, médio ou longo prazo. Todavia, a diversificação é muito importante para se obter rentabilidade de diferentes fontes, e assim, diminuir os riscos de mercado e proporcionar a possibilidade de ganhos mais interessantes. Já para investidores mais conservadores, esse talvez não seja o mercado mais vantajoso, pois às vezes o capital investido fica desvalorizado por longos períodos de tempo, e não se pode descartar também as possibilidades de perca, por ser um ativo que oscila bastante, dessa forma, se o investidor vender com o preço abaixo do que comprou, haverá prejuízo.

\section{REFERÊNCIAS}

AZEVEDO, H.. Bitcoins chegam ao Recife. Paço Alfandega, 2017.

BASILIO, A.. Tudo o que você já consegue comprar com bitcoin no Brasil e no mundo. Época Negócios, 2017.

$B B C$. Por que a falta de mulheres investindo em bitcoins pode ser um mau sinal. BBC, 2018.

BITCOIN. Perguntas Frequentes. Bitcoin, 2015.

CAGLIARI, A.. Pela primeira vez em sete anos número de milionários cai no mundo. Folha, 2019.

CVM. Criptoativos. Investidor. 2018.

FERREIRA, R.. Google Trends mostra maior interesse em Bitcoin do que em ações pela segunda vez na história. Cointelegraph, 2019.

GUSSON, C.. Brasil é um dos cinco países com maior quantidade de usuários de Bitcoin e criptomoedas do mundo. Criptofacil, 2019.

HENRIQUE, M.. Nasce a Primeira Exchange: A História do
Bitcoin parte 6. Guia do Bitcoin, 2019.

INFOCHAIN. Pesquisa compara todas as exchanges de criptomoedas brasileiras: saiba qual é a melhor. Infochain, 2019.

NUNES, M.. Lojas que aceitam bitcoin: descubra onde pagar com bitcoin. LiveCoins, 2019.

PORTAL DO BITCOIN. Número de carteiras de Bitcoin com mais de 0,1 BTC atinge novo recorde. Portal do Bitcoin, 2019.

ROCHA, G.. Criptomoedas: Definições e porque você precisa saber!. Administradores, 2019.

SIMÕES, H.; LAPORTA, T.. Bitcoin já tem mais que o dobro de investidores da bolsa no Brasil. Globo, 2018.

TANCREDI, F.. Estudo inédito mostra quem é o investidor brasileiro de criptomoedas. CoinTimes, 2018.

ULRICH, F.. Bitcoin a moeda na era digital. São Paulo: Instituto Ludwig Von Mises Brasil, 2014.

A CBPC - Companhia Brasileira de Produção Científica (CNPJ: 11.221.422/0001-03) detém os direitos materiais desta publicação. Os direitos referem-se à publicação do trabalho em qualquer parte do mundo, incluindo os direitos às renovações, expansões e disseminações da contribuição, bem como outros direitos subsidiários. Todos os trabalhos publicados eletronicamente poderão posteriormente ser publicados em coletâneas impressas sob coordenação da Sustenere Publishing, da Companhia Brasileira de Produção Científica e seus parceiros autorizados. Os (as) autores (as) preservam os direitos autorais, mas não têm permissão para a publicação da contribuição em outro meio, impresso ou digital, em português ou em tradução. 